\title{
Comparison of point-of-care hemoglobin meters in anemic patients in perioperative and critical care settings
}

\author{
Alan Chan, BSc $\mathbb{B} \cdot$ Ryan Lett, MD, FRCP
}

Received: 6 February 2019/Revised: 13 May 2019/Accepted: 13 May 2019/Published online: 21 May 2019

(C) Canadian Anesthesiologists' Society 2019

\section{To the Editor,}

While validated measures such as the complete blood count and arterial blood gas analysis are currently the goldstandard, results may take up to 45 min to obtain, during which time a rapidly deteriorating patient's status may have changed, rendering the results irrelevant. This becomes especially problematic in the setting of severe anemia where a clinician must decide whether a blood product transfusion should be performed, incurring the potential risks of organ dysfunction, increased hospital length of stay, immunomodulation, alloimmunization, transfusion reactions, thromboembolism, and death. ${ }^{1}$ Point-of-care and indirect testing of hemoglobin has been proposed as a means of improving patient care in the perioperative and critical care environment.

In recent years, different hemoglobin point-of-caretesting (POCT) devices have emerged, with varied mechanisms of action. EPOC (Epocal Inc., Ottawa, ON, Canada) and i-STAT (Abbott Point of Care Inc., East Windsor, NJ, USA) record a hematocrit and mathematically convert this to hemoglobin. EPOC and iSTAT offer the advantage of also being able to measure blood gases in addition to hemoglobin. Craver et al. reported that patients whose anemia was evaluated using a hematocrit were more likely to receive a blood transfusion, have longer hospital stays, and greater costs associated

\footnotetext{
A. Chan, BSc $(\bowtie)$

University of Saskatchewan College of Medicine, Saskatoon, SK, Canada

e-mail: alc643@mail.usask.ca

R. Lett, MD, FRCP

Department of Anesthesiology, Regina General Hospital, Regina, SK, Canada
}

with care. $^{2}$ Alternatively, HemoCue (HemoCue, Ängelholm, Sweden) directly hemolyses erythrocytes and uses photometry to report hemoglobin. There is insufficient information to recommend the use of one device over another. There are currently no recommendations regarding limitations on point-of-care devices, and clinicians are left to evaluate and choose the device that best suits their needs. We performed a comparison study to determine which of these three popular hemoglobin POCT devices was more accurate in measuring hemoglobin in anemic patients compared with the laboratory value.

This study was conducted at the Regina General Hospital and was approved by the Research Ethics Board of the Saskatchewan Health Authority (May 8, 2018: REB18-17). Fifty-eight adult ( $>18$ yr old) patients with a central venous or arterial line who recorded a hemoglobin level less than $100 \mathrm{~g} \cdot \mathrm{L}^{-1}$ by the hematology laboratory were enrolled in the study. All hemoglobin readings were obtained simultaneously within a 20-min time window, and were all done within a two-hour time frame of the blood collection. All three samples recorded were then compared with the gold-standard hematology laboratory analyzer value.

Bland-Altman analysis for HemoCue showed a bias of 1.73 and limits of agreement of -8.44 to 11.91 . EPOC showed a bias of -11.37 and limits of agreement of -50.07 to 27.34. i-STAT showed a bias of 2.41 and limits of agreement of -30.61 to 35.44 (Figure A, B, and C). Deming regression analysis of HemoCue displayed a coefficient correlation of 0.8403 , a -1.8 bias $(-2.1 \%)$, and a standard deviation of 5.1. EPOC showed a coefficient correlation of -0.0896 , bias of $11.6(12.8 \%)$, and standard deviation of 19.5. i-STAT showed a coefficient correlation of -0.169 , bias of $-1.3(-1.6 \%)$, and standard deviation 18.3 . 

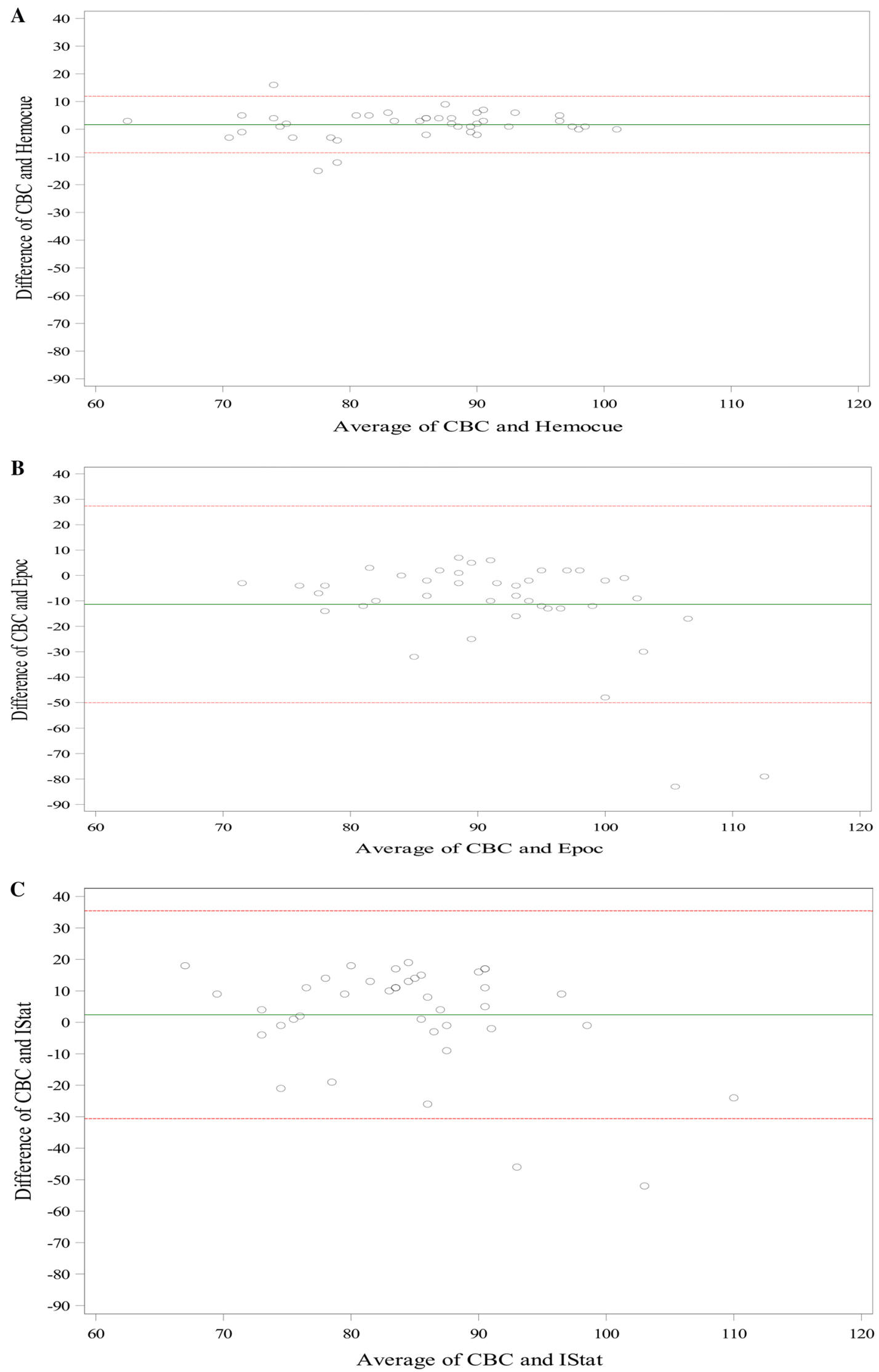
4 Figure A) Bland-Altman plots comparing point-of-care hemoglobin estimates from HemoCue, B) EPOC, and C) i-Stat devices with laboratory hemoglobin standard

Since the landmark TRICC trial by Hébert et al., clinicians have followed a more restrictive transfusion protocol, where transfusion is recommended at hemoglobin concentrations below $70 \mathrm{~g} \cdot \mathrm{L}^{-1}$, and hemoglobin concentration should be maintained between $70 \mathrm{~g} \cdot \mathrm{L}^{-1}$ and $90 \mathrm{~g} \cdot \mathrm{L}^{-13}$. Our study found that, at this range, HemoCue had a stronger correlation to the hematology laboratory analyzer than EPOC and i-STAT and is the most accurate point-of-care testing device for hemoglobin.

Acknowledgements The authors of this study thank the University of Saskatchewan College of Medicine for funding and authorization of the study, SCHAAN Healthcare Products Inc. for the generous donation of HemoCue cuvettes, STARS Air Ambulance for loaning the i-STAT and cartridges during the completion of the study, as well as Siemens AG for supplying the EPOC cartridges.

Conflicts of interest None declared.
Editorial responsibility This submission was handled by Dr. Gregory L. Bryson, Deputy Editor-in-Chief, Canadian Journal of Anesthesia.

Funding This work was funded by the University of Saskatchewan's College of Medicine.

\section{References}

1. Goodnough LT. Risks of blood transfusion. Crit Care Med 2003; 31(12 Suppl): S678-86.

2. Craver C, Belk $K W$, Myers GJ. Measurement of total hemoglobin reduces red cell transfusion in hospitalized patients undergoing cardiac surgery: a retrospective database analysis. Perfusion 2018; 33: 44-52.

3. Hébert PC, Wells G, Blajchman MA, et al. A multicenter, randomized, controlled clinical trial of transfusion requirements in critical care. Transfusion Requirements in Critical Care Investigators, Canadian Critical Care Trials Group. N Engl J Med 1999; 340: 409-17.

Publisher's Note Springer Nature remains neutral with regard to jurisdictional claims in published maps and institutional affiliations. 